\section{DETERMINACIÓN DE UN ÍNDICE DE RENDIMIENTO ACADÉMICO GENERAL PARA MEDIR EL RIESGO DE DESERCIÓN UNIVERSITARIA}

DETERMINATION OF A GENERAL ACADEMIC PERFORMANCE INDEX TO MEASURE THE RISK OF UNIVERSITY DROPOUT

GUSTAVO DEVINCENZI GRICELA ROHDE MARÍA LILIANA BONAFFINI MARTA GIRAUDO ANALÍA PICCINI

Facultad de Ciencias Económicas/Facultad de Ingeniería/Facultad de Arquitectura Universidad Nacional del Nordeste ARGENTINA

Aunque las fuerzas no alcancen, Es loable la osadía: Basta en las grandes empresas Aspirar a conseguirlas. Virgilio

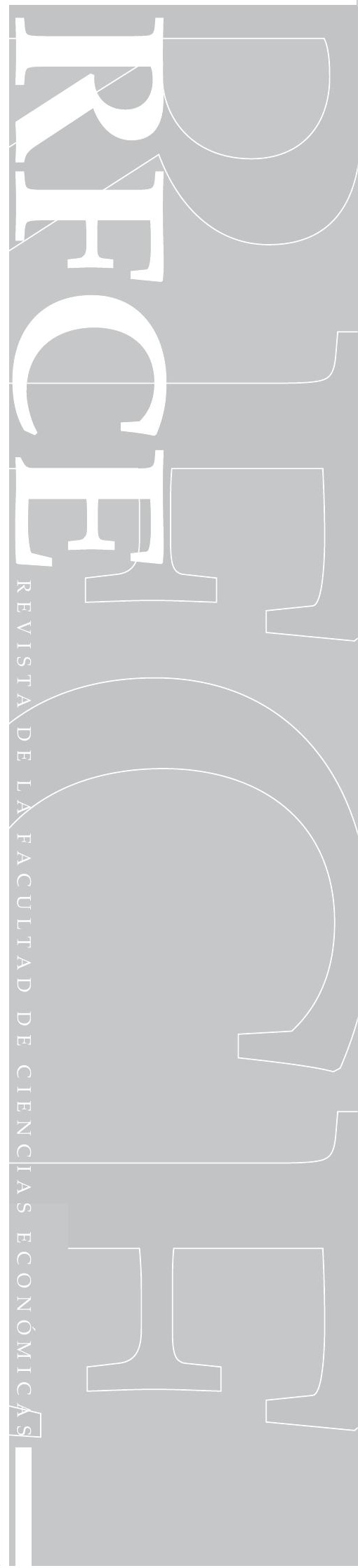




\begin{tabular}{lr}
\hline $\begin{array}{l}\text { GUSTAVO DEVINCENZI - GRICELA ROHDE - MARÍA LILIANA BONAFFINI - MARTA GIRAUDO - ANALÍA PICCINI } \\
\text { DETERMINACIÓN DE UN ÍNDICE DE RENDIMIENTO ACADÉMICO GENERAL... } \\
\text { Págs. 109- 121. DOI: } h \text { ttp://dx.doi.org/10.30972/rfce.0203257 }\end{array}$ \\
\hline
\end{tabular}

\title{
RESUMEN
}

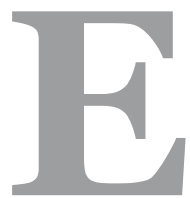

1 rendimiento académico es un fenómeno de múltiples relaciones y complejidades, donde actúan variables subjetivas, históricas y sociales, entre otras; su expresión en las calificaciones obtenidas por el alumno, lo identifican con objetividad.

En este trabajo se presentan los resultados de una investigación efectuada con el objeto de detectar, en forma temprana, situaciones de vulnerabilidad en los alumnos de la Facultad de Ingeniería de la Universidad Nacional del Nordeste, que conlleven a una posible deserción o significativo retraso en sus estudios, a través de la determinación de un índice de Rendimiento Académico General (RAG), buscando una zona crítica que permitiría detectar alumnos con situaciones académicas vulnerables. Se abordó el estudio y construcción de índices a partir de una metodología cuantitativa, bajo el enfoque de investigación desarrollado desde la perspectiva teórica, destacando la importancia de la medición a través de procedimientos estadísticos. Para conseguir el objetivo planteado para este trabajo, se construyeron índices utilizando la situación de cursada y las calificaciones de cada alumno de la población, obteniendo la información del Sistema de Información Universitaria (SIU-Guaraní). Este estudio permitió determinar un intervalo, dentro del cual el valor del RAG, brindó la posibilidad de identificar el grupo de alumnos que debería recibir las acciones remediales/tutoriales, por parte de la unidad académica para disminuir las deserciones y el desgranamiento.

Palabras clave: rendimiento académico, vulnerabilidad, indicadores.

\begin{abstract}
Academic performance is a phenomenon involving multiple links and complexities where subjective, historical and social variables interact altogether, such as student's grades as an expression for identifying it objectively. This paper presents results of a research carried out with the aim of detecting in early stage vulnerable position in students in the School of Engineering at National North-Eastern University which would lead to a possible abandonment or at least a significant delay in their career through a General Academic Performance Index (RAG) determination that searches a critical area and could detect vulnerable academic positions in some students. Study and rate construction was addressed employing a quantitative methodology under a research approaching developed from a theoretical point of view which highlights the importance of reading results through statistical procedures. In order to achieve the objective proposed for this investigation, rates were made by employing current position and grades for each student in the demos, obtaining data through the University Information System (SIUGUARANI). This study allowed thus determination of an interval within which value of RAG
\end{abstract}


showed the possibility to identify the group of students who should receive remedial/tutorial action by Academic Unit to diminish abandonment and flailing of them.

Keywords: academic performance, vulnerability, rate.

\section{1.-INTRODUCCIÓN}

En el marco del proyecto denominado Aplicación de Métodos Matemáticos para Evaluar la Eficiencia y la Vulnerabilidad de los Alumnos en los primeros años de Estudios Universitarios, acreditado por Resol. No 155/15 C.S. de la Universidad Nacional del Nordeste, se viene analizando la problemática del rendimiento académico de los estudiantes universitarios desde el año 2015, año de inicio de la investigación.

Si bien el rendimiento académico es un fenómeno de múltiples relaciones y complejidades, donde actúan variables subjetivas, históricas y sociales, entre otras, su expresión en las calificaciones obtenidas por el alumno, lo identifican con objetividad, brindando según Cascón (2000 p.2), con los índices calculados y analizados, un criterio de fiabilidad y validez.

En este trabajo se presentan los resultados de una investigación efectuada con el objeto de detectar, en forma temprana, situaciones de vulnerabilidad en los alumnos, que conlleven a una posible deserción o significativo retraso en sus estudios, a través del cálculo de un índice de Rendimiento Académico General (RAG), buscando determinar alguna zona crítica del mismo. Esta permitiría detectar alumnos con situaciones acadé-micas vulnerables, en las tres carreras de la Facultad de Ingeniería de la UNNE.

Este índice se seleccionó por su pertinencia para el análisis de nuestro objeto de estudio, de un modelo teórico de Luque y Sequi, (2002, pp.5-14), realizando las modificaciones necesarias para su aplicación en la Unidad Académica mencionada. El mismo está constituido por una suma ponderada de otros tres índices, el Rendimiento Integral de Regularidad, el de Aprobación y el de Logro Cognitivo.

Los índices permiten observar y analizar con un criterio objetivo las variaciones en las calificaciones y en la condición de cada alumno, con respecto a un valor máximo teórico.

Para calcularlos se ha utilizado la base de datos que se encuentra en un Sistema de Información Universitaria (en adelante SIU Guaraní), desde el año 2005 hasta el 2017.

En el análisis de estos indicadores surgieron los siguientes interrogantes: ¿Se podrán encontrar valores de RAG que permitan identificar situaciones de vulnerabilidad/criticidad en los alumnos? ¿Qué variaciones ha experimentado el rendimiento académico en las cohortes analizadas?

Las respuestas a estos interrogantes son la columna vertebral de la investigación que se describe en este artículo. 


\section{2.- MATERIALES Y MÉTODO}

\section{1.- Participantes}

De la información existente en el SIU, de la población de alumnos de las cohortes 20052010, se han analizado tres variables, calificación de examen final o de promoción, condición de fin de cursada, y aprobación. Siendo la primera cuantitativa discreta y las otras dos, cualitativas dicotómicas. Asimismo, se han considerado las fechas en que ocurrieron los eventos significativos (inscripción a la carrera, condición de fin de cursada, evaluación final o promoción).

La fecha de corte para el proceso de datos de cada alumno fue de siete años y medio a partir del año académico de inscripción. Este es el valor medio con que se gradúan los alumnos.

La cantidad total de asignaturas de las tres carreras oscila entre cuarenta y cuarenta y cuatro, más un trabajo final, considerado como una asignatura más, que generalmente insume seis o más meses.

Se seleccionó la información disponible para cada alumno, de las trece asignaturas que integran el ciclo común analizado en Ingeniería - UNNE, así como la cantidad total de materias que aprobó en siete años y medio de actividad académica.

Se trabajó con los datos de inscripción a cursada, regularización y aprobación de las ocho materias de primer año: Álgebra y Geometría, Análisis Matemático I, Sistemas de Representación (Módulo I), Fundamentos de Ingeniería, Análisis Matemático II, Física I, Química, Sistemas de Representación (Módulo II) y de cinco de segundo año: Análisis Matemático III, Informática, Estabilidad I, Física II y Física III.

\section{2.- Método}

Se abordó el estudio y construcción de índices a partir de una metodología cuantitativa, bajo el enfoque de investigación desarrollado desde la perspectiva teórica, destacando la importancia de la medición a través de procedimientos estadísticos.

Para conseguir el objetivo planteado para este análisis, se utilizaron índices elaborados para trabajos anteriores por este mismo grupo de investigación (2016), utilizando las calificaciones de cada alumno de la población, los que se describen a continuación:

\subsection{1.-RENDIMIENTO INTEGRAL DE REGULARIZACIÓN}

Para obtenerlo se comenzó con la determinación de un primer rendimiento que vincula la cantidad de veces que regularizó la materia y la cantidad de veces que la cursó para obtener la regularidad, de acuerdo con el Plan de Estudios en los períodos considerados.

$$
\text { Índice materia regularizada }=\frac{[\text { Cantidad de veces que regularizó la materia }]}{\text { Cantidad de veces que cursó la materia }]}
$$


Estos cálculos se realizaron para cada alumno, en cada una de las 13 asignaturas consideradas, en el período de siete años y medio ya mencionado.

La sumatoria de los índices de todas las materias, nos da la eficiencia total de regularización por alumnos:

$$
\text { Eficiencia total de regularización }=\sum_{\mathrm{j}=1}^{\mathrm{n}} \frac{\mathrm{NR}_{\mathrm{j}}}{\mathrm{NI}_{\mathrm{j}}}
$$

Siendo:

$\mathrm{j}$ : asignatura

NRj: cantidad de veces que el alumno regularizó la asignatura j

NIj: cantidad de veces que el alumno se inscribió para cursar la asignatura j

A esta sumatoria la dividimos por la cantidad de materias que teóricamente debería haber cursado el alumno (MTR) para obtener el rendimiento de regularidad:

$$
\text { Rendimiento de regularidad (RR) }=\frac{\sum_{j=1}^{n} \frac{N R_{j}}{N I_{j}}}{M T R}
$$

Sin embargo, no se debe considerar con igual rendimiento a un estudiante que regularizó las trece materias en los términos previstos por el Plan de Estudios que a aquel que demoró más tiempo en hacerlo. Por este motivo se introduce un coeficiente de ajuste (CA):

$$
\mathrm{CA}=\frac{\left[\begin{array}{c}
\text { Cantidad de meses teóricos para regularizar las } \\
\text { materias del período considerado }
\end{array}\right]}{\left[\begin{array}{c}
\text { Cantidad de meses reales utilizados para } \\
\text { regularizar las materias del período considerado }
\end{array}\right]}
$$

Por lo tanto, el "Rendimiento Integral de Regularización (RIR)" se obtuvo de la siguiente manera:

$$
\mathrm{RIR}=\mathrm{RR} . \mathrm{CA} \frac{\sum_{j=1}^{n} \frac{N R_{j}}{N I_{j}}}{M T R} \cdot \mathrm{CA}
$$

RIR: Rendimiento integral de regularización

RR: Rendimiento de regularidad

CA: Coeficiente de ajuste por retraso en la carrera

Si un alumno aprueba una materia como libre, se considera que obtuvo también la regularización. 


\subsection{2.- RENDIMIENTO INTEGRAL DE APROBACIÓN (RIA)}

Similarmente a la regularización, se procede con la aprobación de asignaturas.

$$
\text { Rendimiento Aprobación }=\frac{\left[\begin{array}{c}
\text { Cantidad de materias aprobadas } \\
\text { durante el tiempo de permanencia }
\end{array}\right]}{\left[\begin{array}{c}
\text { Cantidad de materias que teoricamente debería } \\
\text { haber aprobado según su permanencia }
\end{array}\right]}
$$

$$
\text { Índice materias aprobadas }=\frac{1}{\left[\begin{array}{c}
\text { Cantidad de veces que rindió la } \\
\text { materia hasta su aprobación }
\end{array}\right]}
$$

Estos cálculos se realizarían por cada año académico (marzo a febrero del año próximo)

$$
\text { Eficiencia total de aprobación }=\sum_{\mathrm{j}=1}^{\mathrm{n}} \frac{1}{N E R_{j}}
$$

$j$ : asignatura

NERj: cantidad de veces que el alumno rindió la asignatura j para aprobarla.

La relación entre este índice de eficiencia y la cantidad de materias que teóricamente debería el alumno haber aprobado en función de sus a años de permanencia, se resuelve la primera dimensión que denominamos: Rendimiento Integral de Aprobación (RIA)”, cuya expresión matemática se define como:

$$
R I A_{a}=\frac{\sum_{j=1}^{n} \frac{1}{N E R_{j}} \cdot C A}{M T P E_{a}}
$$

Siendo:

$a=$ años de permanencia en la carrera.

$M T P E$ = materias que teóricamente debería el alumno haber Aprobado en rela-ción al Plan de Estudio en sus a años de permanencia.

$C A$ : coeficiente de ajuste por retraso.

El Coeficiente de Ajuste por Retraso obtenido a partir de la relación proporcional entre la duración teórica de la carrera y la permanencia real.

Matemáticamente podemos expresarlo como sigue:

$$
\mathrm{CA}=\frac{\left[\begin{array}{c}
\text { Cantidad de meses teóricos para aprobar las } \\
\text { materias del período considerado }
\end{array}\right]}{\left[\begin{array}{c}
\text { Cantidad de meses reales utilizados para } \\
\text { aprobar las materias del período considerado }
\end{array}\right]}
$$


$\checkmark$ Las promociones se contaron como alumnos que aprobaron la materia.

$\checkmark$ No se tuvieron en cuenta las aprobaciones por equivalencia en otras carreras.

$\checkmark$ No se consideraron los ausentes de las actas de examen.

\subsection{3.- ÍNDICE DE LOGRO COGNITIVO (ILC)}

Se define como la cantidad proporcional promedio de conocimientos, habilidades y destrezas logradas (aprehendidas) por el estudiante respecto del óptimo propuesto para cada materia aprobada, durante el período de permanencia en la carrera. Este valor de proporcionalidad, para cada materia en particular, está representado por la nota de calificación final de aprobación de dicha asignatura.

$$
I L C_{a}=\frac{\sum_{j=1}^{n} \frac{C F A}{n}}{1 O}
$$

Siendo:

$I L C=$ Índice de Logro Cognitivo.

$C F A=$ Calificación Final de cada materia Aprobada.

$n=$ número total de materias aprobadas.

No fueron considerados los ausentes. La escala de calificación es de o a 10. Se aprueba a partir del 6.

\subsection{4.- ÍNDICE DE RENDIMIENTO ACADÉMICO GENERAL}

En función de todo lo expuesto, se puede definir "Rendimiento Académico General (RAG)", como el resultado de la suma ponderada de los índices parciales de Regularización, Aprobación y Logro Cognitivo. Para el mismo se define la siguiente ponderación:

$$
R A G=0,2 R I R+0,5 R I A+0,3 I L C
$$

Este índice tiene un valor que varía entre o y 1, siendo " 1 " el de mayor rendimiento.

Para el procesamiento de estos datos se utilizaron los aplicativos Visual Foxpro (VFP) y MS Excel.

\subsection{5.- OBTENCIÓN DE ÍNDICES PARA BUSCAR LA DETERMINACIÓN DE UNA ZONA O VALOR DE RAG CRÍTICO}

Se diseñó un conjunto de tablas para realizar el cálculo de los índices, efectuando una adecuación de los datos de la unidad académica con la que se trabajó, para luego ingresar a un proceso de generación de dichos índices, con un script desarrollado en VFP, volcados luego a una planilla Excel.

A partir de los datos obtenidos del Sistema Informático SIU-Guaraní, se procedió a la adecuación de los mismos al formato requerido. 
Se trabajó con las siguientes tablas/vistas de ese sistema: sga_actas_cursado (actas de cursada); sga_comisiones (actas de comisiones); sga_det_acta_curs (detalle de actas de cursada); sga_alumnos (datos de alumnos) y vw_hist_academica (historia académica de los alumnos).

Estos datos fueron proporcionados en formato CSV (comma-separated values). Para un mejor proceso se las pasó a formato de base de datos de VFP, mediante trabajos de conversión.

En este nuevo formato, se verificaron consistencias de las tablas, eliminaron duplicados (hubo casos que figuraban como promocionados y luego aprobadas en un Examen final, etc.) y se adecuaron tipos de datos. Para esto se realizaron scripts específicos en VFP.

De este primer proceso se obtuvieron las 4 tablas que se requieren en el proceso posterior de Generación de Indicadores y Análisis estadístico.

Se definieron como cohortes de trabajo desde la 2005 a la 2010 y 7,5 como la cantidad de años para obtener el título o una cantidad de materias aprobadas equivalente (40 en este estudio).

Para obtener un valor o zona de RAG crítico, se dividió la población total estudiada, en dos grupos:

Grupo (A): alumnos que aprobaron cuarenta materias o más en el lapso considerado. Estos alumnos completaron todo el plan de estudios o estaban a muy escasas materias de lograrlo.

Grupo (B): alumnos que no alcanzaron a aprobar cuarenta materias en el período considerado.

El propósito de esta división fue obtener dos conjuntos de alumnos, los que lograron graduarse en el tiempo promedio, y los que no lo hicieron. A partir de allí, se analizaron los valores de RAG obtenidos en estos grupos, con el objeto de verificar la validez del indicador para evidenciar esta diferencia de performance académica, y en particular, si se podían apreciar valores del mismo que pudieran servir para identificar alumnos en situación de abandono o de criticidad académica en el Grupo B.

\subsection{6.- Proceso de Generación de Índices}

Este proceso se realizó mediante un programa escrito en VFP que toma como Inputs las cuatro tablas indicadas, más un archivo de parámetros. A partir de dichos datos se obtuvo:

- Anonimización de los datos. Requisito necesario para su posterior publicación. Se cambia el $\mathrm{N}^{\circ}$ de legajo por un valor secuencial al azar

- Cantidad de materias regularizadas (de las 13 que se toman como base para este estudio)

- Cantidad de veces que se inscribió a cursar cada materia (de las consideradas)

- Cantidad de materias aprobadas, de las que se toman como base para el estudio (esta aprobación No debe ser por equivalencia)

- Cantidad de veces que se inscribió a rendir cada matera (de las consideradas)

- Tiempo utilizado para la regularización y la aprobación de una materia, y comparación 
respecto al que hubiera correspondido en una cursada a tiempo

- Cantidad total de materias aprobadas (aún por equivalencia, de toda la carrera) en la cantidad de años indicada (7,5 en este estudio)

- Cálculo de los Índices (RIR, RIA; ILG, RAG) de cada alumno.

\subsection{7.- Análisis estadísticos}

Estadísticamente se utilizaron medidas de centralización: media aritmética y de dispersión: desvío típico o estándar, como así también los percentiles dentro de las medidas de orden.

La secuencia seguida para este análisis fue la siguiente:

- Separación en dos grupos, conforme cantidad total de materias aprobadas (Grupos A y

B descriptos en 2.2.5)

- La Media Aritmética ( del RAG y la Desviación Estándar ( , por cohor-te y Grupo.

- Análisis de la Media Aritmética, más y menos la desviación estándar, a los efectos de determinar rangos de valores críticos de RAG.

\section{RESULTADOS}

Con los datos obtenidos se confirmó que la media del Grupo A fue superior, en todas las cohortes, respecto a las del Grupo B, como era de esperarse, dada las características de cada grupo.

Posteriormente, se comparó la media menos el desvío estándar del Grupo A, con la media más el desvío estándar del Grupo B. Se apreció que estos valores eran significativamente coincidentes y se procedió a analizar qué percentiles correspondían en cada caso.

Para el Grupo A, se obtuvo la mejor relación con el percentil 10, y para el Grupo B con el percentil 85. Esto indica que el 90\% de los alumnos del Grupo A se encuentran con RAG mayores o iguales a la media menos el desvío estándar de ese grupo, y que el 85\% de los alumnos del Grupo B, se hallan con RAG menores o iguales a la media más el desvío estándar de ese grupo.

La tabla con un resumen de los datos calculados de los grupos se aprecia a continuación:

Tabla 1: Resumen del análisis estadístico de las cohortes 2005-5010

\begin{tabular}{|c|c|c|c|c|c|c|c|c|c|c|c|c|}
\hline Cohorte & \multicolumn{2}{|c|}{2005} & \multicolumn{2}{c|}{2006} & \multicolumn{2}{c|}{2007} & \multicolumn{2}{c|}{ 2008 } & \multicolumn{2}{|c|}{2009} & \multicolumn{2}{|c|}{2010} \\
\hline Grupo & A & B & A & B & A & B & A & B & A & B & A & B \\
\hline$\mu_{\chi}-\sigma_{\chi}$ & 0,66 & 0,33 & 0,60 & 0,33 & 0,58 & 0,32 & 0,64 & 0,31 & 0,59 & 0,32 & 0,61 & 0,29 \\
\hline$\mu_{\chi}+\sigma_{\chi}$ & 0,90 & 0,58 & 0,89 & 0,59 & 0,90 & 0,63 & 0,89 & 0,60 & 0,83 & 0,62 & 0,87 & 0,60 \\
\hline $\mathrm{P}_{10}$ & 0,65 & 0,65 & 0,60 & 0,60 & 0,56 & 0,56 & 0,64 & 0,64 & 0,54 & 0,54 & 0,61 & 0,61 \\
\hline $\mathrm{P}_{15}$ & 0,67 & 0,32 & 0,62 & 0,33 & 0,59 & 0,33 & 0,64 & 0,30 & 0,61 & 0,32 & 0,63 & 0,28 \\
\hline $\mathrm{P}_{85}$ & 0,58 & 0,58 & 0,60 & 0,60 & 0,62 & 0,62 & 0,62 & 0,62 & 0,62 & 0,62 & 0,60 & 0,60 \\
\hline $\mathrm{P}_{90}$ & 0,98 & 0,61 & 0,97 & 0,63 & 0,95 & 0,65 & 0,96 & 0,65 & 0,90 & 0,67 & 0,95 & 0,66 \\
\hline
\end{tabular}

Fuente: elaboración propia. 
Siendo:

$\mu_{\chi}-\sigma_{\chi}$ : la medida poblacional menos el desvío

$\mu_{\mathrm{x}}+\sigma_{\chi}$ : la medida poblacional más el desvío

$P_{10}, P_{15}, P_{85}, P_{90}$ : percentiles

Estos valores están representados gráficamente en los siguientes diagramas de líneas:

Gráfico 1: Valores estadísticos del Grupo A

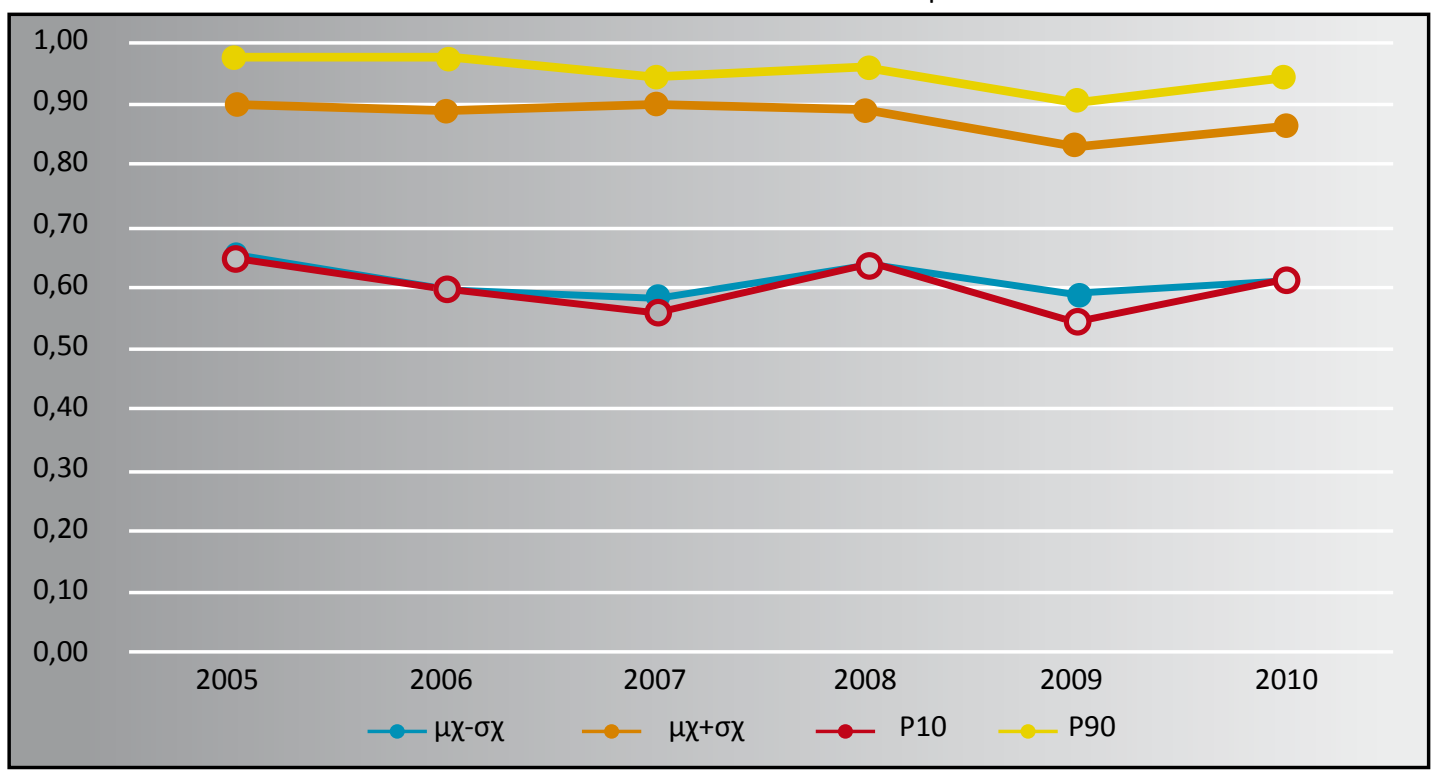

Fuente: elaboración propia

El menor y mayor valor de RAG, obtenido en el Grupo A, para la media menos el des-vío estándar es de 0,58 y 0,66 respectivamente.

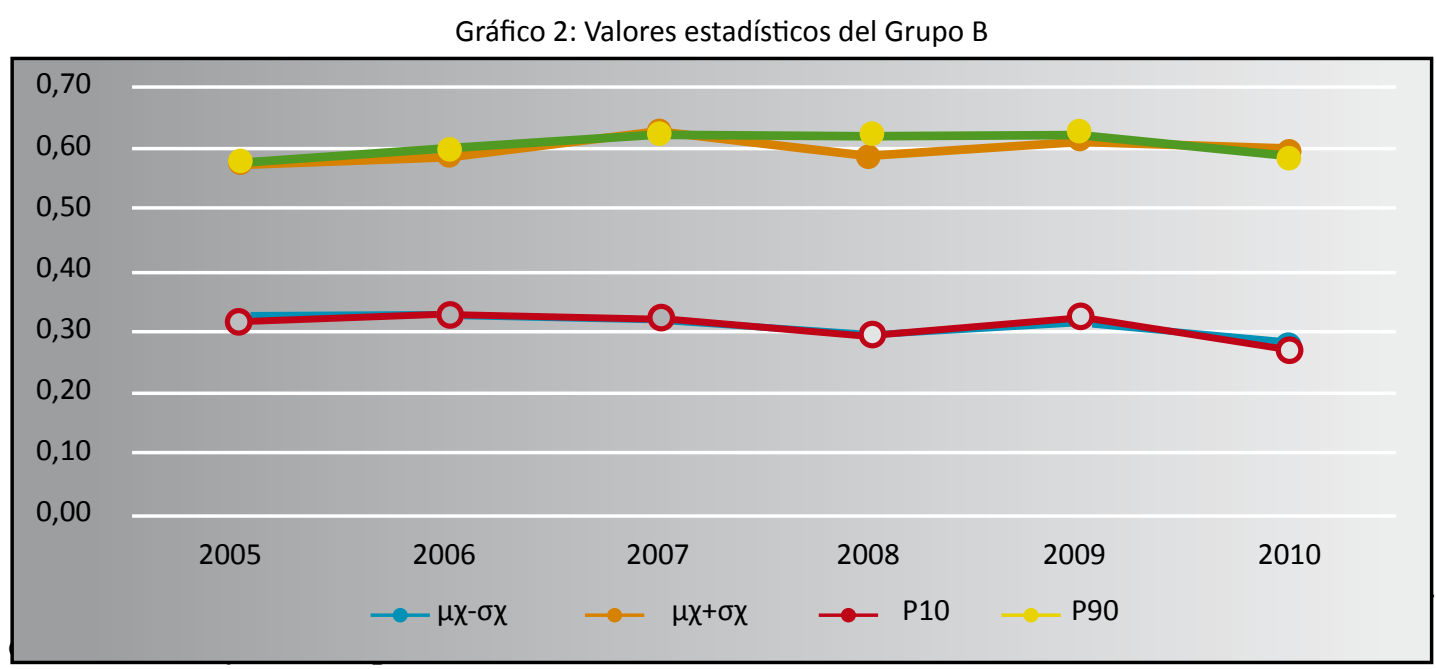

Fuente: elaboración propia 
De esta manera se obtuvo una intersección entre los dos grupos trabajados, hallando un valor crítico para el Índice Académico General, que oscila alrededor del valor 0,6o en todas las cohortes analizadas, como es posible observar en el siguiente gráfico:

Gráfico 3: Variaciones del RAG en todas las cohortes

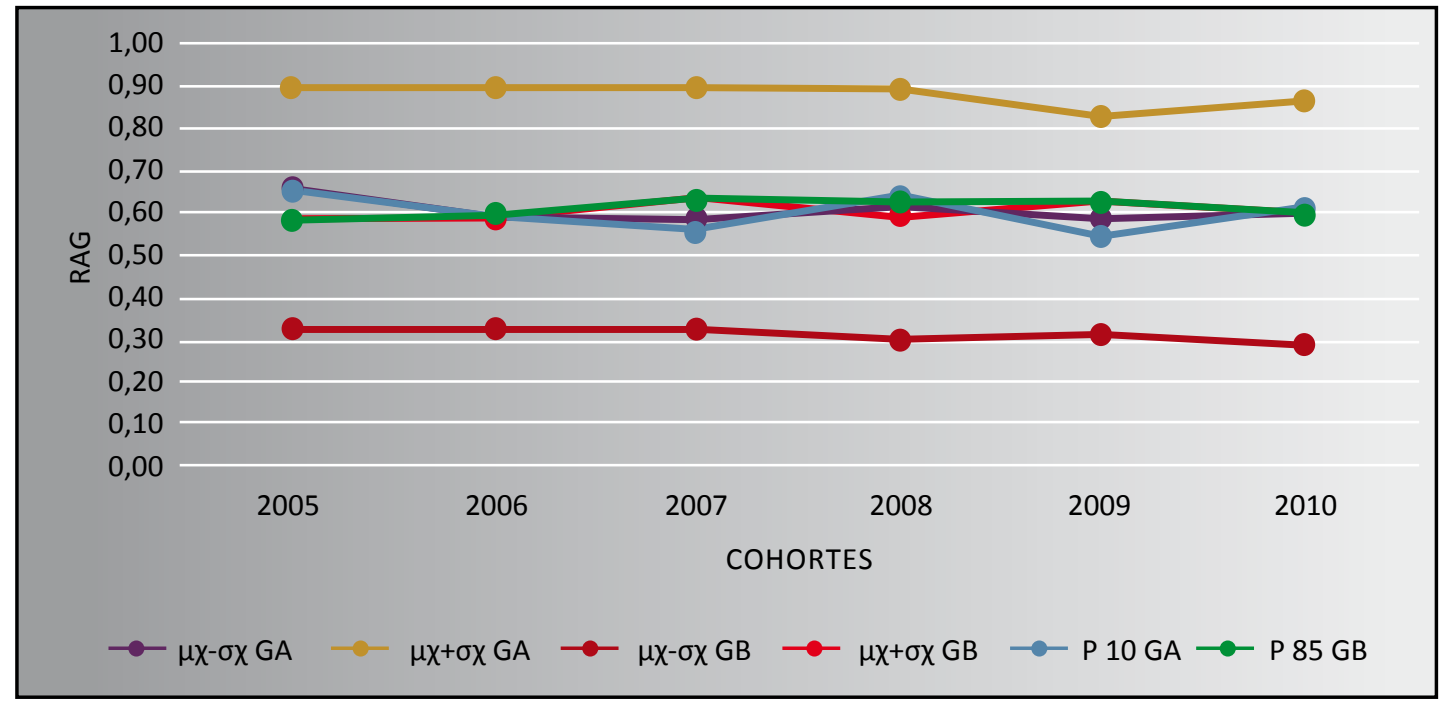

Fuente: elaboración propia

Por debajo de la línea correspondiente a $\mu \chi-\sigma \chi$ GB (media menos el desvío estándar del Grupo B) se encuentran alrededor de 400 alumnos, lo que representa un 30\% de la población total estudiada. Se verificó además que el 95\% de esos alumnos no registran actividad académica por dos años o más, al final del período considerado.

\section{DISCUSIÓN Y CONCLUSIONES}

La presente investigación permitió determinar la franja de alumnos que se encuentran en situación de vulnerabilidad, con riesgo de abandonar sus estudios. Son aquellos cuyos valores de RAG oscilan, entre la media más el desvío y la media menos el desvío del grupo de alumnos que no alcanzaron a aprobar cuarenta materias en el período considerado.

Este estudio aporta información temprana (dentro del ciclo común) sobre el rendimiento académico de los alumnos, y permite inferir el intervalo de variación del RAG, dentro del cual se encuentra el grupo de alumnos que debería recibir las acciones remediales/tutoriales, que la unidad académica viene desarrollando para disminuir las deserciones. 


\section{REFERENCIAS BIBLIOGRÁFICAS}

Cascón, Inocencio V. (2000). Análisis de las calificaciones escolares como criterio de rendimiento académico. En Colegio Público Juan García Pérez. Recuperado de: https:// campus.usal.es/ inico/investigacion/jornadas/jornada2/comun/c17.html

Devincenzi, G.; Rohde, G.; Bonaffini, M.; Bernaola, G.; Giraudo, M., (2016). La medición de la eficiencia y el rendimiento académico de alumnos de los primeros años universitarios. En XXXI Jornadas Nacionales de Docentes de Matemática de Facultades de Ciencias Económicas y Afines. Villa Mercedes. San Luis. Argentina.

Luque, Emilio y Sequi, Juan R. (2002) Modelo teórico para la determinación del rendimiento académico general del alumno, en la Enseñanza Superior. Congreso Regional de Ciencia y Tecnología. NOA 2002. Secretaría de Ciencia y Tecnología. Universidad Nacional de Catamarca. Argentina.

\section{COMO CITAR ESTE ARTÍCULO:}

DEVINCENZI, Gustavo - ROHDE, Gricela - BONAFFINI, María Liliana - GIRAUDO, Marta - PICCINI, Analía. Determinación de un índice de rendimiento académico general para medir el riesgo de deserción universitaria. Revista de la Facultad de Ciencias Económicas - UNNE. Número 20, otoño 2018, ISSN 1668 - 6365. Págs. 109 - 121. DOI: http://dx.doi. org/10.30972/rfce.0203257

\section{CURRICULUM VITAE}

\section{Gustavo H. Devincenzi}

Ingeniero en Construcciones/Ingeniero Civil. Especialista en Docencia Universitaria. Profesor Titular con Dedicación Exclusiva de la Cátedra: "Informática" de la Facultad de Ingeniería de la UNNE y Profesor Titular con Dedicación Simple de la Cátedra "Procesamiento de Datos" de la Facultad de Ciencias Económicas de la UNNE. Docente Investigador, Categoría III. gdevin@ing.unne.edu.ar 


\section{Gricela Alicia Rohde}

Profesora en Matemática y Cosmografía. Especialista en Investigación Educativa y Magíster en la Enseñanza de la Matemática. Profesora Titular con Dedicación Exclusiva de la Cátedra "Matemática I", Profesora Adjunta de la Cátedra “Matemática II”. Directora del Departamento de Matemática de la Facultad de Ciencias Económicas de la UNNE. Docente Investigadora, Categoría IV.

grohde@eco.unne.edu.ar

\section{María Liliana Bonaffini}

Contadora Pública. Especialista en Docencia Universitaria. Profesora con Mayor Dedicación en el cargo de Auxiliar Docente de $1^{\circ}$ Categoría en la Cátedra de Costos de la Facultad de Ciencias Económicas de la UNNE. Docente Investigadora, Categoría III.

mbonaffini@eco.unne.edu.ar

\section{Marta Beatriz Viviana Giraudo}

Ingeniera en Construcciones. Magister en la Enseñanza de la Matemática. Profesora Titular de Álgebra y Geometría, Facultad de Ingeniería. Profesora Titular de Ciencias Básicas, Facultad de Arquitectura, UNNE. Directora del Instituto de Matemática Facultad de Ingeniería, Categoría $\mathrm{V}$ de investigación.

marta_giraudo@yahoo.com.ar

\section{Analía Piccini}

Arquitecta. Especialista en Docencia Universitaria. Profesora Titular de Estadística Educativa y Profesora Adjunta de Estadística en la Facultad de Humanidades. Profesora Adjunta de Estadística Aplicada en la Facultad de Criminalística y Profesora Adjunta de Ciencias Básicas en la Facultad de Arquitectura. apapiccini@gmail.com 